\title{
Dry Arthroscopy Distal Radioulnar Joint and Foveal Insertion: Surgical Technique
}

\author{
Jeff Ecker, BMedSc (hons), FRACS ${ }^{1,2}$ Courtney Andrijich, BSc, MA ${ }^{1}$ \\ ${ }^{1}$ Jeff Ecker Clinic, Bethesda Hospital, Claremont, Western Australia, \\ Australia \\ ${ }^{2}$ Hand and Upper Limb Centre, Claremont, Western Australia, Australia \\ Address for correspondence Courtney Andrijich, BSc, MA, Jeff Ecker \\ Clinic, Bethesda Hospital, 25 Queenslea Dr, Claremont WA 6010, \\ Australia 012 (e-mail: Courtney.Andrijich@ecker.com.au).
}

J Wrist Surg 2022;11:2-5.

Abstract
Keywords
- dry arthroscopy
- distal radioulnar joint
- triangular
fibrocartilage
- foveal insertion
- wrist

Background Arthroscopy of the distal radioulnar joint is considered to be difficult to perform. At this time the integrity of the foveal insertion is indirectly evaluated with a hook test. If a hook test is positive it is inferred that the foveal insertion is torn or incompetent.

Description of Technique The ideal way to evaluate the foveal insertion is by direct visualization and probing. In order to do this, arthroscopic examination of the distal radioulnar joint and foveal insertion is required. The article describes how to reliably perform "dry" arthroscopy of the distal radioulnar joint and foveal insertion using a $1.9 \mathrm{~mm}$ arthroscope to accurately assess the triangular fibrocartilage complex and foveal insertion.

Patient and Methods A total of 169 dry DRUJ arthroscopies were performed by the primary author between January 2018 and February 2021.

Results Using this technique, the foveal insertion was successfully visualized in 168 cases (99\%).

Conclusion Dry arthroscopy of the DRUJ is a reliable technique to evaluate the integrity of the foveal insertion.
Arthroscopic examination of the distal radioulnar joint (DRUJ) can be difficult. ${ }^{1,2}$ The foveal insertion of the triangular fibrocartilage complex (TFCC) has been reported to be visible in $57 \%$ to $64 \%$ of cases. ${ }^{1,2}$ Nakamura et al was able to view the foveal insertion using wet arthroscopy in 170 of 196 (87\%) wrists. ${ }^{3}$ Inability to view the foveal insertion was attributed to synovial tissue obscuring the view. ${ }^{3}$ Currently, the most common way of determining the integrity of the foveal insertion is an indirect assessment using the "hook" test. It is inferred that a positive "hook" test is pathognomonic of a tear or deficiency of the foveal insertion of the TFCC. ${ }^{4}$ The ability to see and probe the foveal insertion is the most accurate way to evaluate its integrity. This paper describes the technique of "dry" arthroscopic surgery of the DRUJ using a 1.9-mm arthroscope. Using dry arthroscopic techniques, it is possible to remove the synovial tissues which can obscure the arthroscopic view of the foveal insertion. Once the technique of

received

March 28, 2021

accepted after revision

June 15, 2021

published online

July 28, 2021

dry arthroscopy of the DRUJ is mastered, it is possible to confidently view and probe the foveal insertion.

\section{Anatomy}

The arthroscopic portals used to perform dry arthroscopy of the DRUJ are the $3 / 4$ portal, 6R portal, dorsal DRUJ portal, and the volar DRUJ portal.

The $3 / 4$ portal is just distal and ulnar to the tubercle of Lister (-Fig. 1). The 6R portal is immediately radial to the extensor carpi ulnaris (ECU) (-Fig. 1). The dorsal DRUJ portal is immediately proximal to the $6 \mathrm{R}$ portal (-Fig. 1). The 6R and dorsal DRUJ portals are longitudinal incisions which are so aligned that, if open surgery is required, they can be connected, and the incision line extended. The volar DRUJ portal is the soft spot immediately volar to the base of the ulnar styloid (-Fig. 1). It can be felt in all positions of

(c) 2021. Thieme. All rights reserved. Thieme Medical Publishers, Inc., 333 Seventh Avenue, 18th Floor, New York, NY 10001, USA
DOI https://doi.org/ $10.1055 / \mathrm{s}-0041-1732414$. ISSN 2163-3916. 


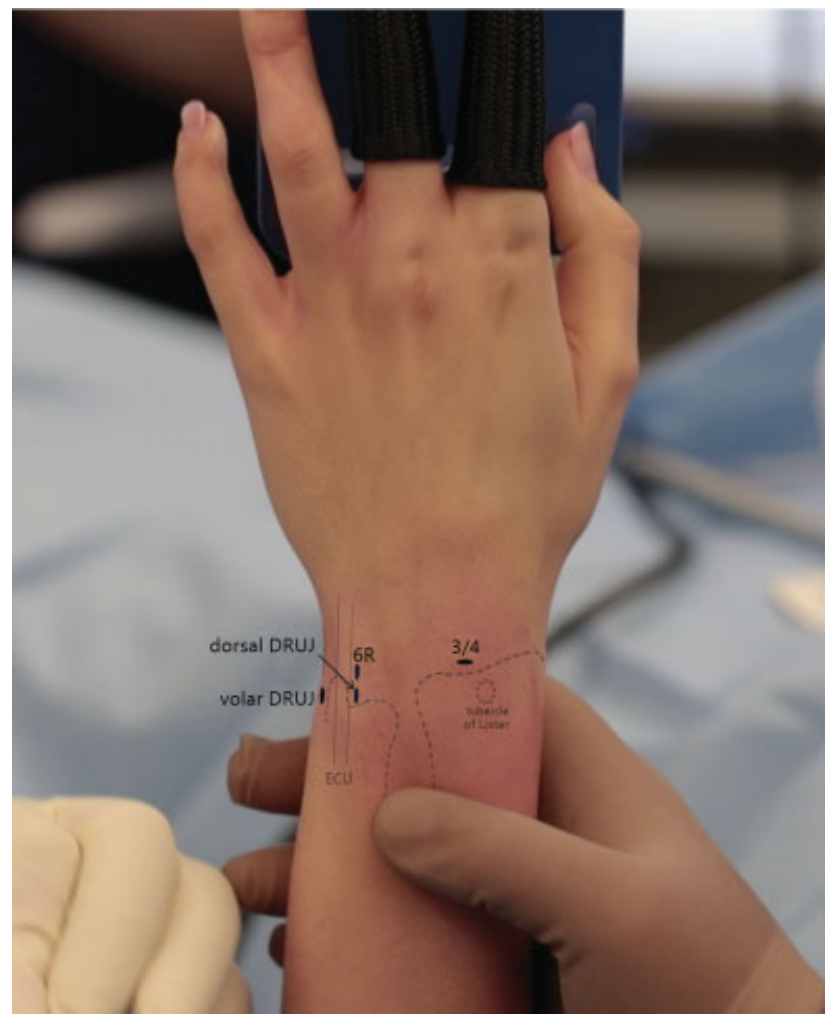

Fig. 1 Surface anatomy showing placement of arthroscopic portals.

pronation and supination, and it is easier to appreciate with the forearm in maximum supination. The foveal insertion is immediately deep to the ulnar styloid, and its insertion is dorsal to the entry point of the volar DRUJ portal.

The anatomy and the importance of the foveal insertion has been described by Nakamura et al. ${ }^{3,5}$

\section{Indications and Contraindications}

There are two indications for dry arthroscopy of the DRUJ. The first is diagnostic to evaluate the possible causes of ulnarsided wrist pain. The second is therapeutic.
The contraindications for an elective dry arthroscopy of the DRUJ are superficial infections, abrasions of the skin in the region of the arthroscopic portals, and patients who are not optimally fit for elective surgery.

Another relative contraindication for dry arthroscopy of the DRUJ is a surgeon who is not trained or experienced in the technique. There is a learning curve associated with this procedure. When surgeons are learning how to perform this procedure, it can be difficult, and there is a risk of failure, damage to the joint, injury to the neurovascular structures and damage to the $1.9-\mathrm{mm}$ diameter arthroscope. For this reason, it is recommended that surgeons do not perform this procedure unless they are supervised by an experienced surgeon who knows how to perform this technique or they have acquired the skills by attending cadaver arthroscopy workshops.

\section{Technique}

\section{Setup}

Finger traps are applied proximal to the proximal interphalangeal joints (PIPJ) of the index and middle fingers, and traction is applied using a traction tower.

\section{Procedure}

This arthroscopic procedure is performed using a "dry" technique. Irrigation is not used (-Video $\mathbf{1}$ ).

\section{Video 1}

The surgical technique of dry arthroscopy of the triangular fibrocartilage complex (TFCC) and foveal insertion. Online content including video sequences viewable at: https://www.thieme-connect.com/ products/ejournals/html/10.1055/s-0041-1732414.

(1) Insert a 1.9-mm arthroscope in the $3 / 4 \operatorname{portal}$ (-Fig. 2A). (2) Create a 6R portal ( - Fig. 2A). This is done under direct vision while viewing through the $3 / 4$ portal (-Fig. 2A). An

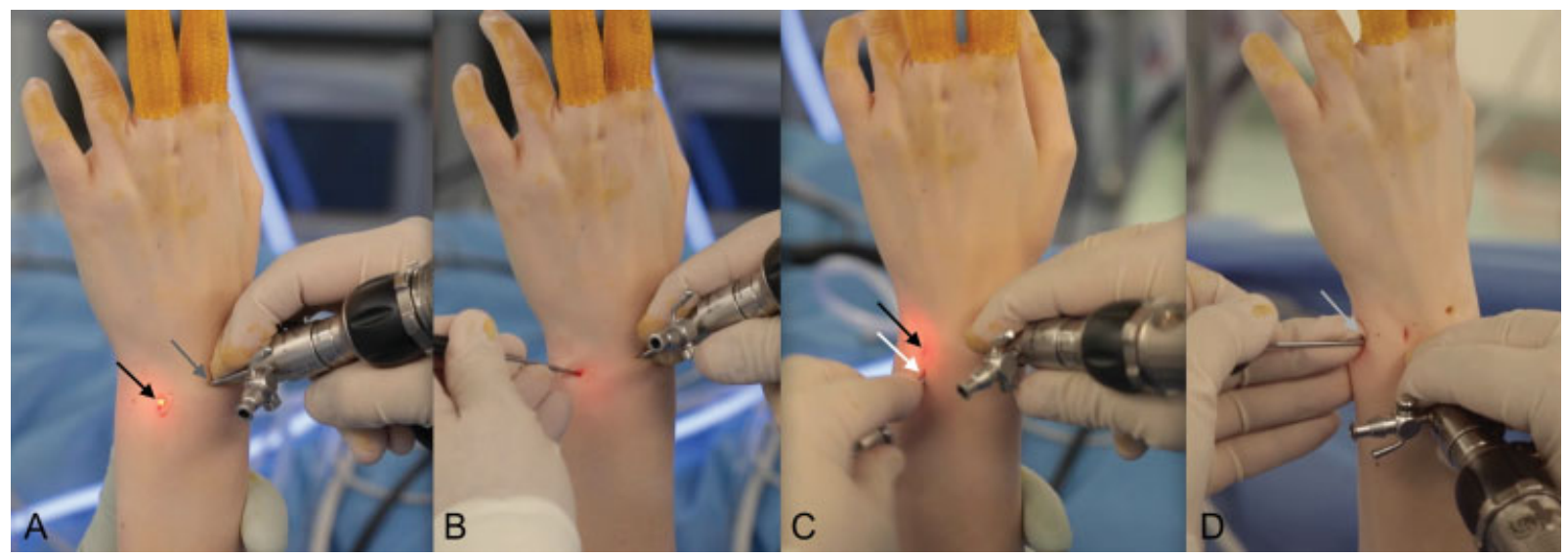

Fig. 2 (A) Arthroscope in 3/4 portal (dark grey arrow) and creation of 6R portal (black arrow). (B) Arthroscope in 3/4 portal and small joint hook probe in $6 \mathrm{R}$ portal to perform a trampoline test and "hook" test. (C) Arthroscope in the 3/4 portal. Obturator and cannula in dorsal DRUJ portal (white arrow). (D) Arthroscope in dorsal DRUJ portal. Shaver in volar DRUJ portal (light gray arrow). 


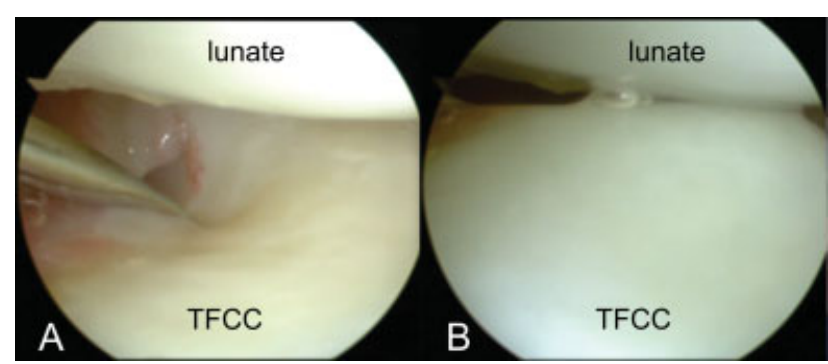

Fig. 3 (A, B) Positive hook test. A small joint hook probe displacing the TFCC (labeled) distally showing the TFCC making contact with the articular surface of the lunate (labeled). TFCC, triangular fibrocartilage complex.

18-gauge needle is inserted to identify the correct location of the $6 \mathrm{R}$ portal and entry into the radiocarpal joint. Once the location has been correctly identified a longitudinal stab incision is made in the skin with a number 11 scalpel blade. Small mosquito forceps are then inserted through the $6 \mathrm{R}$ portal to enlarge the portal.

(3) Examine the radiocarpal surface of the TFCC, using a hook probe. A small joint hook probe is inserted into the 6 R portal ( - Fig. 2B), and the tension of the TFCC assessed using a "trampoline" test and a "hook" test (-Fig. 3A, B).

(4) While viewing from the $3 / 4$ portal, an 18 -gauge needle is inserted proximally and in alignment with the $6 \mathrm{R}$ portal. The distal head of the ulna is felt with the $18 \mathrm{G}$ needle to confirm that the needle is correctly placed proximal to the TFCC. Care needs to be taken not to insert the needle into the substance of the TFCC. Once the correct orientation and position has been established with the 18 -gauge needle, a longitudinal portal incision is made in alignment with the $6 \mathrm{R}$ portal, and mosquito forceps are inserted into the same space proximal to the TFCC. Once again correct placement can be confirmed by feeling the articular surface of the distal ulna and displacing the TFCC distally under direct vision, using the scope in the $3 / 4$ portal. A second obturator and cannula is then inserted into the dorsal DRUJ portal while visualizing the distal surface of the TFCC with the scope in the $3 / 4$ portal (-Fig. 2C). The correct placement is again confirmed by feeling the articular surface of the distal ulna and displacing the TFCC distally under direct vision through the $3 / 4$ portal ("ghost" sign) (-Fig. 4A, B). The 1.9-mm arthroscope is then removed from the $3 / 4$ portal and inserted into the cannula in the dorsal DRUJ portal.

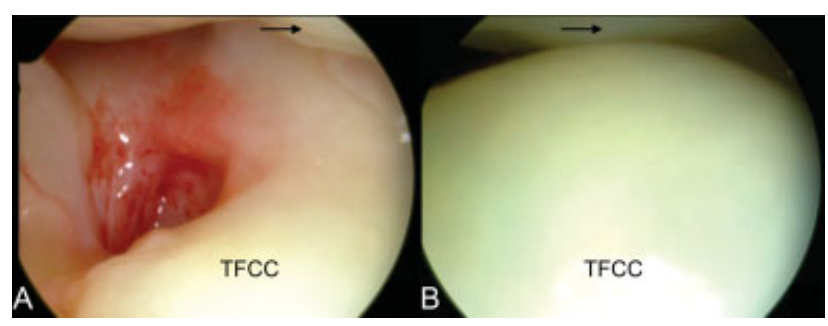

Fig. 4 (A, B) Positive ghost sign with the TFCC (labelled) elevated to touch the articular surface of the lunate (black arrow). TFCC, triangular fibrocartilage complex.
(5) The $1.9-\mathrm{mm}$ arthroscope is then rotated 90 degrees directed toward the ulnar styloid.

(6) The arthroscope is then slowly retracted dorsally while viewing ulnarly in the direction of the fovea to visualize the foveal insertion. It is emphasized that this maneuver involves millimeter increments. To achieve this controlled micromovement of the arthroscope, it is recommended that the scope is held in such a way that the index finger, middle finger, and thumb can retract the arthroscope while the ring finger is in contact with the wrist. In most cases, it is not possible to reliably assess the foveal insertion, because it is obscured by soft tissue. This soft tissue needs to be removed. The solution is to create a volar DRUJ portal where a $2.7-\mathrm{mm}$ or 3-mm shaver can be inserted to remove the soft tissue under direct vision with the scope in the dorsal DRUJ portal.

(7) Volar DRUJ portal. While viewing with the $1.9-\mathrm{mm}$ arthroscope in the dorsal DRUJ portal, an 18-gauge needle in inserted through the soft spot at the base of the ulnar styloid ( - Fig. 5A, B).

Technical tip: The plane of entry of the 18-gauge needle and instruments into the volar DRUJ portal is at right angles to the long axis of the ulna and volar to the ulnar styloid.

The view of the needle entering the palmar DRUJ portal can be obscured by soft tissue. When it is not possible to see the tip of the needle, triangulation is required. The 18-gauge needle needs to be held in a similar albeit different way to the arthroscope. The middle finger is in contact with the wrist, and the index finger and thumb withdraw and insert the needle in small increments, using the middle finger as a counter pressure point. In this way, it is possible to intuit the contact point of the tip of the arthroscope and the tip of the needle. This is a learned skill, but once acquired, it can be reliably and confidentially performed ( - Video 2 ).

\section{Video 2}

Debridement of the soft tissue to see and feel the foveal insertion. Online content including video sequences viewable at: https://www.thieme-connect. com/products/ejournals/html/10.1055/s-00411732414 .

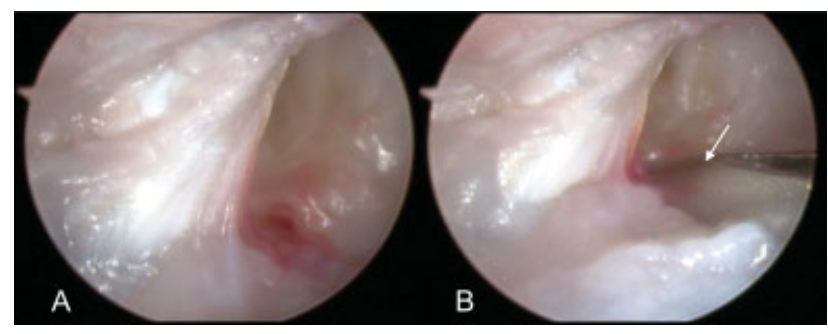

Fig. 5 (A) View of a normal foveal insertion with the arthroscope in the dorsal DRUJ portal. (B) 18-gauge needle (white arrow) in the volar DRUJ portal viewed with the arthroscope in the dorsal DRUJ portal. DRUJ, distal radioulnar joint. 
The needle is then removed and the volar DRUJ portal created using mosquito forceps inserted in the same plane as the 18-gauge needle. Once the tip of the mosquito forceps can be felt and seen, a 2.7- or 3-mm shaver is inserted into the volar DRUJ portal (-Fig. 2D). Only when the tip of the shaver is identified and its anatomical location known can the shaver be used safely to remove the soft tissue, which is obscuring the view of the foveal insertion. The shaver is manipulated dorsally and ulnarly with the blade against the foveal insertion to remove the soft tissue obscuring the view of the foveal insertion. This is done gently and not forcibly. An intact foveal insertion will not be removed by the shaver used in this way. Once the soft tissue has been debrided, the foveal insertion can be seen and probed to evaluate whether it is intact, damaged, or absent.

(8) The scope is then manipulated radially to examine the sigmoid fossa. If there is DRUJ instability, it is relatively easy to see the sigmoid fossa.

(9) Sometimes, it is not possible to clearly see and probe the foveal insertion while viewing from the dorsal DRUJ portal. When this occurs, a second obturator and cannula is inserted into the volar DRUJ portal under direct vision with the scope in the dorsal DRUJ portal. The scope is then changed from the dorsal DRUJ portal to the volar DRUJ portal.

(10) If required, the foveal insertion can be debrided with a shaver inserted into the dorsal DRUJ portal. The foveal insertion can then be probed through the dorsal DRUJ portal.

Technical tip: When first learning how to view the foveal insertion and DRUJ with the scope in the volar DRUJ portal, it can be difficult to orientate. If the surgeon changes position from the dorsum of the wrist and works from the axilla, the technique and orientation remain the same as working with the arthroscope in the dorsal DRUJ portal. Once the surgeon is familiar with using the volar portal, there is no need to change positions.

\section{Closure}

The arthroscopic portals are closed with adhesive tape.

\section{Rehabilitation}

For a diagnostic arthroscopy, the patient begins active and passive excises within their range of comfort immediately after surgery.

\section{Discussion}

In our experience, dry arthroscopic surgery of the DRUJ is highly reliable. Retrospective analysis of 169 dry DRUJ arthroscopies performed by the primary author between January 2018 and February 2021 revealed that the foveal insertion was successfully visualized in 168 cases (99\%). Dry arthroscopy of the DRUJ provides information beyond the resolution of MRI. The limitations of MRI in accurately diagnosing TFCC tears has been previously reported to have a sensitivity ranging from 69 to $76 \%$ and specificity from 41 to $60 \%{ }^{6,7}$ At present, the integrity of the foveal insertion is evaluated by indirect means using the "hook" test. This means the integrity of the foveal insertion is a deduction, based on the presence of a positive hook test and the anatomical knowledge of the foveal insertion. ${ }^{4}$ Using "dry" arthroscopy of the DRUJ, the foveal insertion can be seen and probed from both the volar and dorsal DRUJ portals, so that the integrity and the quality of the foveal insertion is directly assessed. The proximal surface of the TFCC seen in its entirety and the distal articular surface of the head of the ulna and the articular surface of the sigmoid fossa can be examined using this technique.

In our experience, the view achieved using this dry arthroscopic technique is clearer than that obtained using normal saline. Dry arthroscopy also avoids the problem of normal saline extravasating from the joint into the surrounding soft tissues.

The key points to mastering this technique is the use of two obturators and cannulae, so that the obturator can be changed from one portal to another under direct vision. The scope can then be confidently changed to the second portal which is the correct position.

This is an endoscopic microsurgical procedure and requires vision and an awareness of where the tip of the scope and instruments are at all times. It is emphasized that the primary author is an expert in wrist arthroscopy. ${ }^{8}$ In the early stages, while learning, the fragile $1.9-\mathrm{mm}$ scopes can be easily damaged, because the shaver is in close proximity to the scope. However, once the skill is acquired, it is possible to work with the scope and the shaver within millimeters of each other without damaging the scope. Using the technique described, the surgeon can successfully and reliably evaluate the integrity of the foveal insertion in nearly all cases.

Funding
None.

Conflict of Interest

None declared.

\section{References}

1 Yamamoto M, Koh S, Tatebe M, et al. Arthroscopic visualisation of the distal radioulnar joint. Hand Surg 2008;13(03):133-138

2 Yamamoto M, Koh S, Tatebe M, et al. Importance of distal radioulnar joint arthroscopy for evaluating the triangular fibrocartilage complex. J Orthop Sci 2010;15(02):210-215

3 Nakamura T, Matsumura N, Iwamoto T, Sato K, Toyama Y. Arthroscopy of the distal radioulnar joint. Handchir Mikrochir Plast Chir 2014;46(05):295-299

4 Atzei A. New trends in arthroscopic management of type 1-B TFCC injuries with DRUJ instability. J Hand Surg Eur Vol 2009;34(05): 582-591

5 Nakamura T, Takayama S, Horiuchi Y, Yabe Y. Origins and insertions of the triangular fibrocartilage complex: a histological study. J Hand Surg [Br] 2001;26(05):446-454

6 Hahn P, Häusler A, Bruckner T, Unglaub F. [Quality rating of MRI regarding TFCC lesions in the clinical practice]. Handchir Mikrochir Plast Chir 2012;44(05):310-313

7 Schmauss D, Pöhlmann S, Lohmeyer JA, Germann G, Bickert B, Megerle K. Clinical tests and magnetic resonance imaging have limited diagnostic value for triangular fibrocartilaginous complex lesions. Arch Orthop Trauma Surg 2016;136(06):873-880

8 Tang J, Giddins G. Why and how to report surgeons' level of expertise. J Hand Surg [Br] 2000;25:180-182 